\title{
Energy-Balanced Compressive Data Gathering in Wireless Sensor Networks
}

\author{
Cuicui Lv $v^{\mathrm{a}, *}$, Qiang Wang ${ }^{\mathrm{a}, *}$, Wenjie Yan ${ }^{\mathrm{b}}$, Yi Shen ${ }^{\mathrm{a}}$ \\ ${ }^{a}$ Department of Control Science and Engineering, Harbin Institute of Technology, Harbin, China \\ ${ }^{b}$ School of Computer Science and Engineering, Hebei University of Technology, Tianjin, China
}

\begin{abstract}
Compressive Sensing(CS) can use fewer samples to recover a great number of original data, which have a sparse representation in a proper basis. For energy-constrained Wireless Sensor Networks(WSNs), CS provides an effective data gathering approach. Gaussian random matrix satisfies Restricted Isometry Property(RIP) with high probability. The class of matrices are usually selected as the measurement matrix for compressive data gathering in WSNs. However, they are dense, and the computational complexity is higher. On the other side, sparse binary matrix with a fixed number of nonzero entries in each column satisfies RIP-1 property. Due to the higher sparsity, the class of sparse binary matrix is chosen as the measurement matrix in the paper. In order to adapt to the dynamic change of network topology, we design a mobile agent based compressive data gathering algorithm (MA-Greedy), where each sensor node is uniformly visited in $M$ measurements. Coefficient of Variation $(C V)$ is proposed to evaluate the balance of energy consumption. The numerical experiments show the proposed algorithm is superior to other algorithms(i.e. non-CS, plain-CS, Hybrid-CS, and Distributed Compressive Sparse Sampling(DCSS)) in terms of energy balance. Moreover, we discover the performance of reconstructing sparse zero-one signals by sparse binary matrix, which is used in the proposed MA-Greedy algorithm, is better than that by Gaussian random matrix when Basis Pursuit(BP) algorithm is used for signal recovery.
\end{abstract}

Keywords:

compressive sensing, wireless sensor networks, sparse binary matrix, mobile agent, energy balance

\section{Introduction}

Wireless sensor networks(WSNs) consist of a large number of sensor nodes. Their task is to periodically gather data and transmit the data to sink node by wireless communication Yick et al. (2008). Sensor nodes are usually powered by batteries. The calculation ability and communication ability are limited Anastasi et al. (2009). Once deployed, they may receive little maintenance. In the complex and inaccessible environment monitoring, it is hard to replenish energy. How to gather the data in an energy-efficient way becomes the most urgent question for energy-constrained WSNs.

In conventional sampling approaches, the signals are first uniformly sampled at or above the Nyquist rate, which requires a minimum number of samples, and compressed during the transmission. The approach can eliminate the redundant information and reduce traffic burden. But most samples are thrown away in the data compression, wasting tremendous time and energy. A novel framework for signal acquisition Zhao and Huang (2012), Compressive Sensing(CS), has emerged and received significant attention from researchers. In CS, sampling and compression are simultaneous. It only samples a part of data which aren't thrown away in the data compression. Compared with Shannon's sampling theory, CS enables a potentially large reduction in the number of samples and the computation costs.

\footnotetext{
${ }^{*}$ Corresponding author

Email address: Ivcuicuixuchuan@163.com(Cuicui Lv); wangqiang@hit.edu.cn (Qiang Wang)
}

When the signals are sparse or compressible in a suitable basis or dictionary, CS theory can be used to recover the signals from a small set of linear, non-adaptive measurements Candès (2006); Donoho (2006); Baraniuk (2007). Since the signal$\mathrm{s}$ are linearly projected on the measurement matrix for measurements, the computational complexity of encoding is lower. Besides, encoding and decoding are independent. Decoding is operated at sink node, further reducing energy consumption of sensor nodes. So CS provides an efficient approach for data gathering in WSNs.

In the burst events monitoring Xie et al. (2011), compared with multitudinous sensor nodes, the number of sensor nodes that have monitored the burst events happening is very sparse. CS can be applied to sparse data gathering in WSNs. In the paper, we mainly address the energy balance problem of compressive data gathering in WSNs. Because the network topology changes dynamically, compressive data gathering algorithm is required to have good robustness. Mobile agent is intelligent and it doesn't need to keep all the sensor nodes synchronized. It is particularly attractive in this problem. We adopt mobile agent based compressive data gathering approach to transmit the sensed data. The main contributions of the paper are as follows.

(1) We discuss compressive data gathering in WSNs. Dense measurement matrices require all the sensor nodes to participate in data gathering, leading to more energy consumption. We choose sparse binary matrix as the measurement matrix in CS. Because the number of nonzero entries in sparse binary matrix is less than that of zero entries, it leads 
to a significant reduction in computational complexity and storage requirements.

(2) In CS paradigm, we first use mobile agent to gather sparse data in WSNs. Like Minimum Traveling Salesman Problem(MTSP), the path design of mobile agent is a Constrained Minimum Traveling Salesman Problem(CMTSP), which is NP-hard. We put forward a compressive data gathering approach based on greedy algorithm.

(3) The total energy consumption of WSNs is the major measure metric in the existing literatures. There exists some sensor nodes that relay a large number of data from other$s$ in the tree-type structure. Their energy consumption is much more than others. When the energy of these sensor nodes is depleted, the network may be disconnected, which can shorten the network lifetime. That is, the minimum energy consumption doesn't guarantee that energy consumption of each sensor node is more balanced. To evaluate the balance of energy consumption, we propose Coefficient of Variation $(\mathrm{CV})$ and compare the performance of proposed MA-Greedy algorithm with that of other data gathering algorithms.

The remainder of the paper is organized as follows. In Section 2. we review related works about compressive data gathering in WSNs. In Section 3, we give a brief overview of CS, and describe the network model. Coefficient of Variation $(C V)$ in WSNs is defined and mobile agent based compressive data gathering algorithm(MA-Greedy algorithm) is presented in Section 4 In Section 5, we conduct the experiments to demonstrate the performance of MA-Greedy algorithm in terms of energy balance. Section 6 concludes the paper. Table 1 summarizes the notations in the paper.

\section{Related works}

In recent years, the application of CS to data gathering in WSNs has been receiving increasing attention. An early application of CS in WSNs is Bajwa et al. (2007), where a distributed joint source-channel communication architecture was proposed for energy-efficient estimation of sensor field data, and sensor nodes synchronously transmitted the sensed data to sink node in a single hop way. While it doesn't consider how to use CS in multi-hop communication.

For multi-hop networking scenarios, CS in conjunction with routing was exploited in Quer et al. (2009), where random projections of sensed data were transmitted to sink node. Routing matrix was the measurement matrix whose coefficients were generated by the same pseudo-random number generator at the sensor nodes. Unlike the contribution in Quer et al. (2009), Lee et al. (2009) considered the communication cost and presented a centralized, greedy algorithm for energy efficient data gathering in WSNs, but the results showed the effectiveness of CS was limited. Luo et al. (2009) first analyzed traditional data gathering and Compressive Data Gathering $(C D G)$ in the chain-type topology. Then they presented the first complete design to apply CS to sensor data gathering
Table 1: Summary of notations used in the paper

\begin{tabular}{ll}
\hline notations & definitions \\
\hline$G(V, E)$ & the undirected graph $G(V, E)$ \\
$V$ & the set of vertices in $G(V, E)$ \\
$E$ & the set of edges in $G(V, E)$ \\
$s_{0}$ & sink node \\
$s_{i}$ & sensor node with ID $i(1 \leq i \leq N)$ \\
$e_{i j}$ & edge between $s_{i}$ and $s_{j}$ \\
$n e i g\left(s_{i}\right)$ & the neighborhood nodes of $s_{i}$ \\
$x$ & a sparse signal of size $N \times 1$ \\
$\Psi$ & the orthonormal basis \\
$\Theta$ & the coefficient vector \\
$k$ & the sparsity of $x$ \\
$M$ & the number of measurements \\
$\Phi_{M \times N}$ & the measurement matrix of size $M \times N$ \\
$y$ & the measurement vector \\
$d$ & the number of nonzero entries \\
$I N_{i}$ & the interest node of mobile agent with ID $i$ \\
$E_{T x}$ & energy consumption of transmitting the information \\
$E_{R x}$ & energy consumption of receiving the information \\
$d_{0}$ & the given threshold \\
$L$ & the size of the information \\
$E_{\text {elec }}$ & the parameter of transmitting/receiving circuit \\
$E_{a m p}$ & the parameter of the amplifier in transmitting circuit \\
$n$ & the path loss factor \\
$R$ & communication radius \\
$C V$ & coefficient of variation \\
$\sigma$ & standard deviation \\
$\bar{E}$ & the average energy consumption of WSNs \\
$\hat{x}$ & the reconstruction signal of $x$ \\
$\varepsilon$ & relative error \\
\hline &
\end{tabular}

in large-scale WSNs. Each sensor node transmitted $M$ messages. Tree-type structure was chosen to transmit the random projections. When sink node received $M$ weighted sums of random projections, reconstruction algorithm was used to recover the original signal. Directly CS coding on each sensor node was called plain CS aggregation(plain-CS) in Xiang et al. (2013), where Xiang et al. (2013) proposed a data aggregation technique called Hybrid CS aggregation(Hybrid-CS). Only when the outgoing data flows of sensor nodes were no less than the number $M$ of measurements, sensor nodes started $\mathrm{C}$ $\mathrm{S}$ coding. Minimum Spanning Tree(MST) and data aggregation were jointed to minimize the total energy consumption. Fazel et al. (2011) introduced the application of Random Access Compressed Sensing(RACS) in underwater environmen$\mathrm{t}$ monitoring. RACS employed random sensing for the sampling procedure and a simple random access for the channel access phase to prolong the network lifetime. Chen and Wassell (2011) proposed an energy-efficient signal acquisition approach for monitoring 1-D environmental information. Random sampling and the Sampling Rate Indicator(SRI) feedback were introduced. Random sampling could trade off the randomization and computational complexity. SRI feedback maintained the reconstruction quality and reduced energy consumption. Mamaghanian et al. (2011) quantified the potential of CS for energy-efficient ECG data acquisition and compression on resource-constrained Wireless Body Sensor Networks(WBSN) platforms. The implementation of Gaussian random matrix was too complex, and time consuming. It was certainly not a real- 
time task for the MSP430. They explored three different methods, where sparse sensing matrix was superior in terms of execution time.

Dense random matrices are chosen as the measurement matrix in most available literatures. As described above, the implementation of dense random matrices is too complex and time consuming. Because of lower complexity and higher sparsity, sparse binary matrix is exploited as the measurement matrix. Intelligent mobile agent is introduced for compressive data gathering in WSNs.

\section{Problem description and network model}

In the section, we first review CS theory, and analyze two classes of CS measurement matrices, dense random matrices and sparse random matrices. Finally mobile agent based network model is given.

\subsection{CS Overview and design of sparse binary matrix}

Suppose a WSN consists of $N$ sensor nodes and one sink node. Each sensor node has a random ID. WSN is abstracted to an undirected graph $G(V, E)$, where $V$ and $E$ denote the set of vertices and edges, respectively. $V$ is written as $V=\left\{s_{0}, s_{1}, \ldots, s_{i}, \ldots, s_{N}\right\}$, where $s_{0}$ denotes sink node and $s_{i}$ is sensor node with ID $i(1 \leq i \leq N)$. If two sensor nodes $s_{i}$ and $s_{j}$ can communicate with each other, edge $e_{i j}$ exists. $E$ is expressed as $E=\left\{e_{i j} \mid 1 \leq i<j \leq N\right\}$.

The data reading of $s_{i}$ is $x_{i}$, and the data readings $x$ of $N$ sensor nodes are a column vector $x=\left[x_{1}, x_{2}, \ldots, x_{i}, \ldots, x_{N}\right]^{T}$ of size $N \times 1$. If $x$ has a sparse or compressible representation in the orthonormal basis $\Psi \in \mathbb{R}^{N \times N}, x$ can be expressed as

$$
x=\Psi \Theta
$$

where $\Theta \in \mathbb{R}^{N \times 1}$ is the coefficient vector of $x$. $\|\Theta\|_{0}$ denotes the number of nonzero entries in $\Theta$. If $\|\Theta\|_{0}=k(k \ll N), x$ is $k$ sparse.

The linear measurement vector $y=\left[y_{1}, y_{2}, \ldots, y_{i}, \ldots, y_{M}\right]^{T}$ is acquired by

$$
y=\Phi x=\Phi \Psi \Theta
$$

where $\Phi \in \mathbb{R}^{M \times N}(M \ll N)$ is the measurement matrix denoted as $\Phi_{M \times N}$. If $\Phi_{M \times N} \Psi$ satisfies RIP, it is sufficient to recover the signal $x$ from a small number of linear measurements. Furthermore, the number $M$ of measurements needs to meet the condition in Eq.(3), where $c$ is a positive constant.

$$
M \geqslant c \cdot k \cdot \log (N / k)
$$

Dense random matrices, such as independent and identically distributed Gaussian random matrix or Bernoulli matrix, satisfy RIP with overwhelming probability and they are usually selected as the measurement matrix $\Phi_{M \times N}$ in WSNs. However, it is complex to implement them. In addition, most entries in dense random matrices are non-zero. This requires a large number of sensor nodes to participate in compressive data gathering, leading to more energy consumption.
Another class of matrices are called sparse random matrices. Wang et al. (2007) showed sparse random matrices were sufficient for data recovery, and the performance was comparable to the optimal $k$-term approximation. Sparse binary matrix is in this category. Li and Qi (2013) analyzed the relation between expander graph and sparse binary matrix in detail. Extensive experiments on both synthetic and real data sets showed CS recovery accuracy by sparse binary matrix outperformed existing sparse random matrices in Wang et al. (2007).

For the design of sparse binary matrix $\Phi_{M \times N}$, we randomly choose $d$ locations in each column of $\Phi_{M \times N}$. Let the entries on these locations be one and the other entries are zero. Such matrices have a one-to-one correspondence with the adjacency matrices of fixed left degree expander graph, and they can be constructed explicitly by expander graphs $\mathrm{Xu}$ and Hassibi (2007); Jafarpour et al. (2009).

\subsection{Mobile agent based network model}

Each row of $\Phi_{M \times N}$ expresses a path to sink node. In Wang et al. (2007), each row is assigned to a projection node. For a WSN with $N$ sensor nodes, each sensor node is selected as the projection nodes with probability of $M / N$ in $M$ measurements. The different projection nodes lead to different energy consumption, so the selection of projection nodes can affect energy consumption. Mobile agent is a special kind of software or computer program. It can migrate between nodes in a network to perform a task(or tasks) autonomously and intelligently Chen et al. (2007). Different from the approach in Wang et al. (2007), the paper uses mobile agent for compressive data gathering in WSNs.

Suppose sparse binary matrix is $\Phi_{M \times N}$. Sink node assigns mobile agents with different IDs to each row in $\Phi_{M \times N}$. If the entries in the $i$ th $(1 \leq i \leq M)$ row are $\left[\phi_{i 1}, \phi_{i 2}, \phi_{i 3}, \ldots, \phi_{i j}, \ldots, \phi_{i N}\right]$, mobile agent with ID $i$ leaves sink node and visits the interest nodes $I N_{i}$ whose coefficients $\phi_{i j}(1 \leq j \leq N)$ are nonzero. The nodes in $I N_{i}$ are visited by the proposed MA-Greedy algorithm in Section 4. When mobile agent with ID $i$ migrates to an interest node $s_{j}$, it acquires the data reading $x_{j}$ of $s_{j}$ and gets a projection $\phi_{i j} \cdot x_{j}$. Then the mobile agent will carry the weighted sums $\sum \phi_{i j} \cdot x_{j}$ of projection to the next interest node, which hasn't been visited. After all the interest nodes in the row are visited, mobile agent carries the measurement $y_{i}=\sum_{j=1}^{N} \phi_{i j} \cdot x_{j}$ to sink node. Through $M$ measurements, sink node gets the measurement vector $y=\left[y_{1}, y_{2}, \ldots, y_{i}, \ldots, y_{M}\right]^{T}$. According to CS reconstruction algorithms, sink node can recover the original data readings $x$ of sensor nodes from the measurement vector $y$.

\section{Compressive data gathering algorithm}

In the section, we define Coefficient of Variation $(C V)$ in WSNs. Describe mobile agent based compressive data gathering algorithm(MA-Greedy algorithm) and analyze its time complexity. 


\subsection{Coefficient of Variation $(C V)$}

Energy consumption $E_{T x}(i)$ for $L$ bits information transmitted by $s_{i}$ over the distance $d_{i}$ is given in Eq.(4).

$$
E_{T x}(i)=\left(E_{\text {elec }}+E_{\text {amp }} \times d_{i}^{n}\right) \times L
$$

where $E_{\text {elec }}$ and $E_{a m p}$ are the parameters of transmitting/receiving circuit and the amplifier in transmitting circuit Abdulla et al. (2012). $n$ is the path loss factor, ranging from 2 to 4 . The value of $n$ depends on the given threshold $d_{0}$. If $d_{i}$ is less than $d_{0}$, the free space channel model( $d_{i}^{2}$ power loss) is used. Otherwise, the multipath channel model $\left(d_{i}^{4}\right.$ power loss $)$ is used Heinzelman et al. (2002).

$E_{R x}(i)$ is energy consumption of $s_{i}$ receiving $L$ bits information.

$$
E_{R x}(i)=E_{\text {elec }} \times L
$$

The total energy consumption of $s_{i}$ is $E_{\text {Total }}(i)$.

$$
E_{\text {Total }}(i)=E_{T x}(i)+E_{R x}(i)
$$

In order to evaluate the difference of the total energy consumption among sensor nodes, we define Coefficient of Variation $(C V)$ in WSNs.

$$
\begin{gathered}
C V=\frac{\sigma}{\bar{E}} \\
\sigma=\sqrt{\frac{1}{N} \sum_{i=1}^{N}\left(E_{\text {Total }}(i)-\bar{E}\right)^{2}} \\
\bar{E}=\frac{1}{N} \sum_{i=1}^{N} E_{\text {Total }}(i)
\end{gathered}
$$

where $\sigma$ is standard deviation, and $\bar{E}$ is the average energy consumption of WSNs.

If the value of $C V$ is smaller, the total energy consumption of each sensor node is more concentrated, and the difference of the total energy consumption among sensor nodes is smaller. In other words, the smaller the value of $C V$ is, the more balanced the total energy consumption among sensor nodes is.

\subsection{Compressive data gathering algorithm}

In general, the undirected network topology graph $G(V, E)$ isn't a complete graph. In order to make mobile agents move from one node to anther nodes, the degree of each node (i.e. the number of link edges to the node) is at least two. In the condition, the network may be disconnected. The network connectivity must be guaranteed such that mobile agents can return to the original point after visiting their interest nodes. We confirm the network connectivity by two methods. One method is the construction of spanning trees in $G(V, E)$. If $G(V, E)$ contains a spanning tree, $G(V, E)$ is connected. Otherwise, $G(V, E)$ is disconnected. The other one is Breadth First Search(BFS). If every node can be visited by BFS, $G(V, E)$ is connected. Or $G(V, E)$ is disconnected. When $G(V, E)$ is disconnected, other nodes are added or the communication radius $R$ is increased until $G(V, E)$ is connected.
If $G(V, E)$ is connected and the degree of each node is at least one, there must be Minimum Spanning Tree(MST). There will be no loops in MST unless some edges are added. In this case, mobile agents can also visit their interest nodes. But a large number of nodes will be visited for many times, which results in more energy consumption. So we require that the degree of each node is at least two.

Theorem 1. If $G(V, E)$ is connected and the degree of each $n$ ode is at least two, mobile agents can return to sink node after visiting the interest nodes by the loops.

Proof. The number of sensor nodes and the link edges in WSNs are finite, so $G(V, E)$ is finite. There must exist a tremendous path $P$ in $G(V, E)$ West and West (2001). Let $u$ be a terminal vertex in $P$. Then all neighborhood nodes of $u$ must be in $P$. Because the degree of $u$ is at least two, the neighborhood node $v$, making the link edge $e_{u v}$ between $u$ and $v$ not be in $P$, of $u$ exists. $e_{u v}$ and the path from $v$ to $u$ in $P$ form a loop. In other words, if the degree of each node is at least two, $G(V, E)$ contains at least a loop. In the monitored area, the interest nodes are distributed uniformly. Mobile agents leave sink node to visit them and finally return to sink node. Their path design needs the loops. However, the isolated loops may exist. So $G(V, E)$ is required to be connected. Under the two conditions, mobile agents can return to sink node after visiting their interest nodes.

Theorem 2. If sparse binary matrix is $\Phi_{M \times N}$ and mobile agents with different IDs visit their interest nodes, their path design is NP-hard.

Proof. Each column of $\Phi_{M \times N}$ has $d$ nonzero entries. If $N$ entries in the $i$ th row of $\Phi_{M \times N}$ are nonzero and $N$ sensor nodes can communicate with each other, the path design of mobile agent with ID $i$ is in close proximity to Travelling Salesman Problem(TSP), which is NP-hard. This is a particular case of the path design of mobile agent. Generally speaking, mobile agent only visits a few of sensor nodes rather than all sensor nodes. But if these sensor nodes can communicate with each other, the path design of mobile agent is also similar to TSP. Otherwise, other sensor nodes that aren't the interest nodes are added to connect the network. In a word, the path design of mobile agents can be regarded as CMTSP. Because the path design of mobile agents in the particular case, where all the interest nodes can communicate with each other, is NP-hard, CMTSP is NP-hard.

When the network topology is a complete graph, Qi et al. studied the dynamic path planning of mobile agents, and put forward Local closest first(LCF) and global closest first(GCF) Oi and Wang (2001). In GCF, the current node chose the closest node to the center node as the next node in each iteration, leading to path inflections and extra energy consumption. Its performance was worse than LCF. As described in section III, each mobile agent must visit its interest nodes. How to visit the interest nodes is a Constrained Minimum Traveling Salesman Problem(CMTSP), and CMTSP is NP-hard. Inspired by 


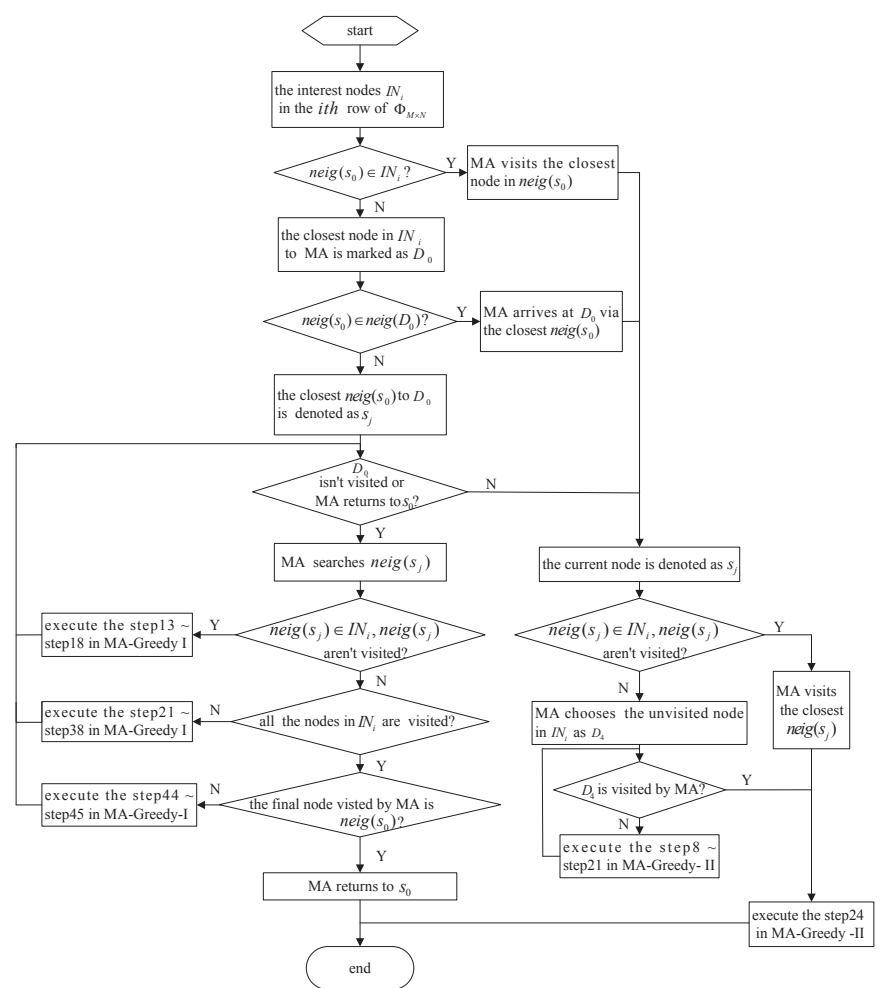

Fig. 1. Flow chart of MA-Greedy-I and MA-Greedy-II

LCF, the paper adopts greedy algorithm to design the paths of mobile agents.

Mobile agent based compressive data gathering algorithm (MA-Greedy) contains two parts. The first part is MA-GreedyI, where mobile agent with ID $i$ visits the closest node in $I N_{i}$. If mobile agent can directly move to the closest interest node by LCF, the first part ends. Otherwise, the closest node in $I N_{i}$ is selected as a new destination node $D_{0}$ and the neighborhood nodes neig $\left(s_{0}\right)$ of $s_{0}$ conducts the step10 step49 until mobile agent arrives at $D_{0}$. In the process, a part of interest nodes may be visited. If the unvisited interest nodes exist, the second part MA-Greedy-II is conducted. In MA-Greedy-II, we mainly design the path of mobile agent migrating from an interest node to the other interest nodes. When all the interest nodes are visited and the final node in the path is sink node $s_{0}$, MA-Greedy-II ends. Flow chart is shown in Fig 1.

In MA-Greedy algorithm, if all the neighborhood nodes of $s_{j}$ are visited once and the interest nodes which haven't been visited exist, the visited nodes are required to backtrack. When the visited node can communicate with the interest node closest to $s_{j}$, the backtracking process is stopped. Otherwise, FloydWarshall algorithm is used to make mobile agent move from $s_{j}$ to the closest interest node.

\subsection{Algorithm analysis}

In the best case, neig $\left(s_{0}\right) \in I N_{i}$ and sensor nodes in $I N_{i}$ can communicate with each other. Mobile agent with ID $i$ can visit the closest interest node in each visit. For each mobile agent, the time complexity of each measurement is the same

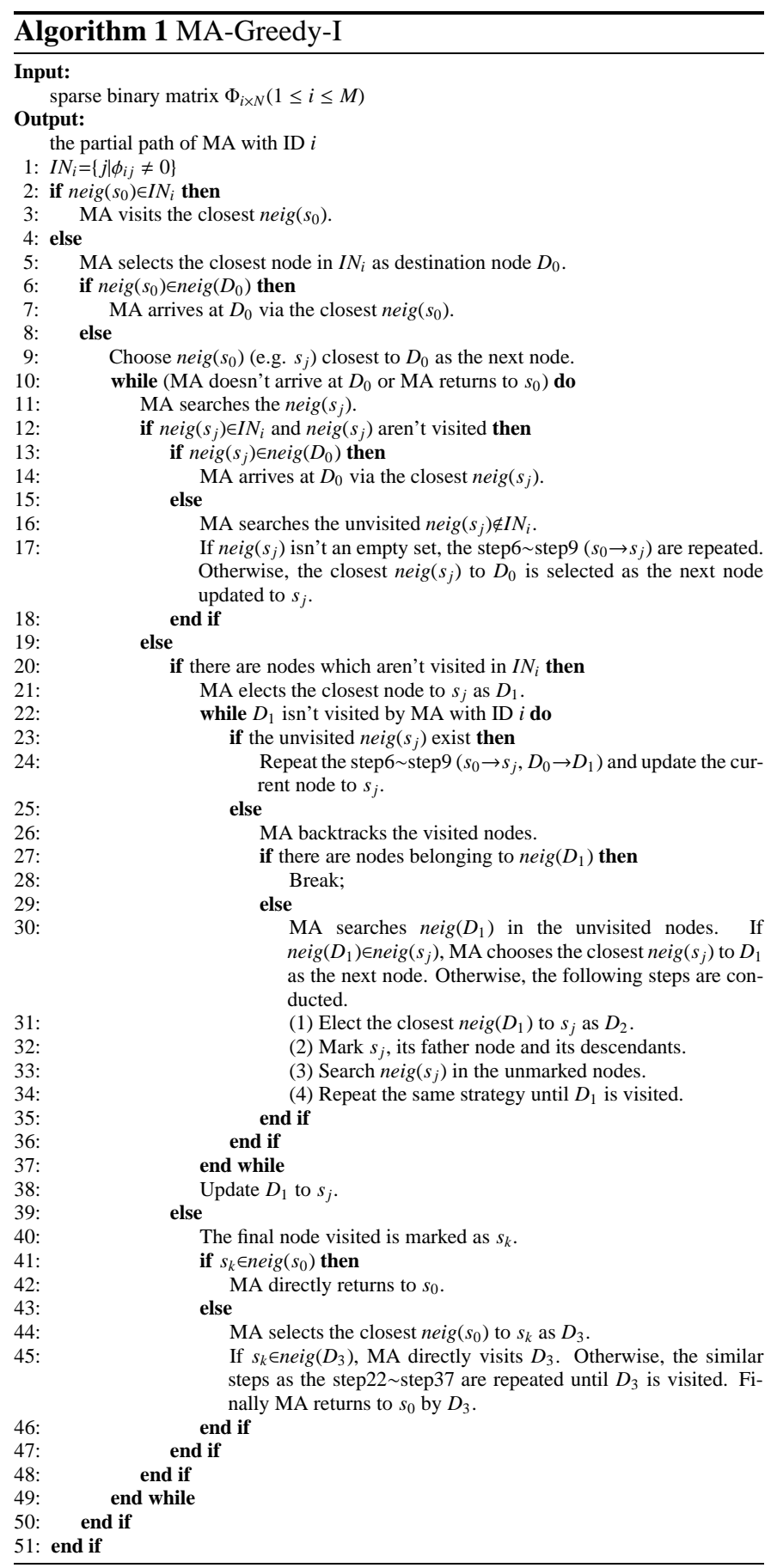




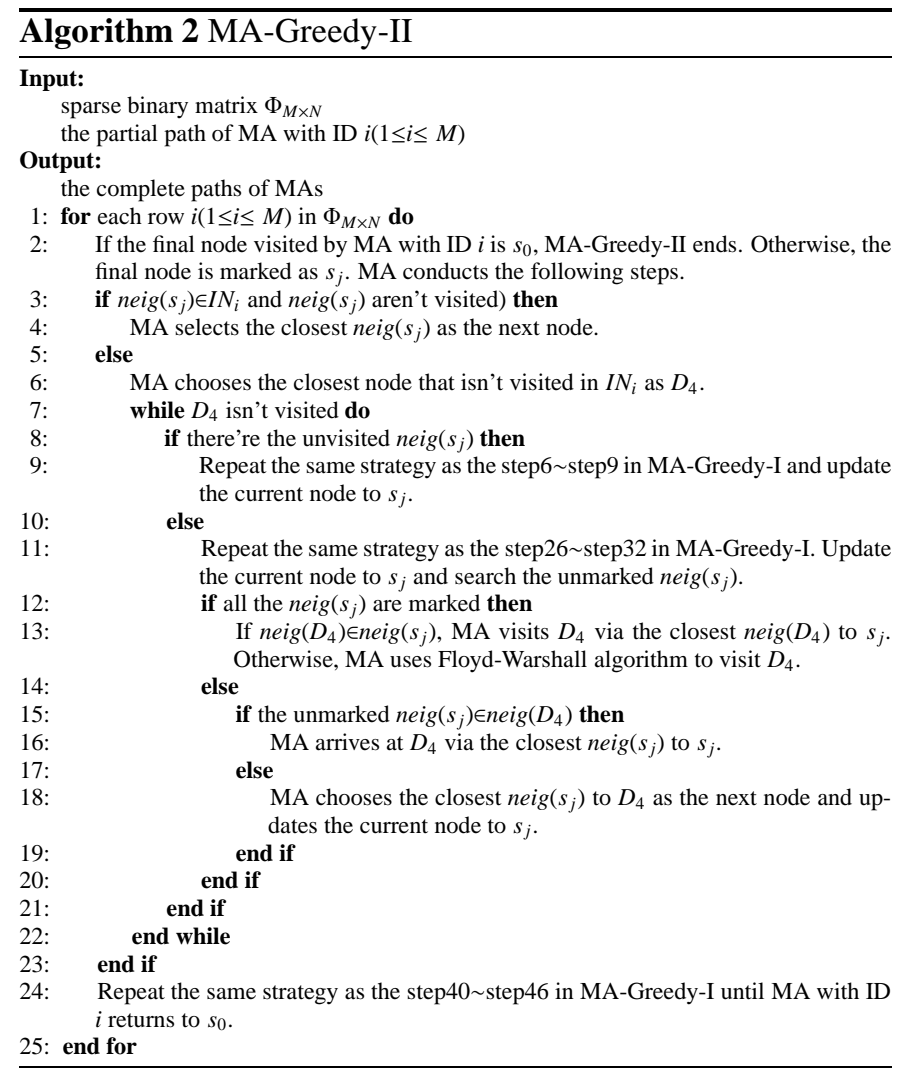

as sort algorithm. MA-Greedy algorithm contains $M$ measurements. The average time complexity of MA-Greedy algorithm is $O\left(M \tau_{\text {max }} \log \tau_{\max }\right)$, where $\tau_{\max }$ is the maximal number of the nodes in $I N_{i}$.

In the worst case, all the nodes in $I N_{i}$ can't communicate with each other, and other nodes which aren't the interest nodes are added in the network. All the nodes participate in data gathering and Floyd-Warshall algorithm is used. The time complexity of MA-Greedy algorithm is $O\left(M N^{3}\right)$.

We give an example of MA-Greedy algorithm. Construct a sparse binary matrix $\Phi_{50 \times 100}$, where $d$ is 4 . According to MAGreedy-I, we determine the entries in $I N_{1}, I N_{27}, I N_{37}$ and $I N_{47}$. Then mobile agents visit the nodes in $I N_{i}$ by the paths designed by MA-Greedy algorithm. As shown in Fig, 2 , the magenta solid dots are the nodes in $I N_{i}$. The cyan solid dots are the other nodes that aren't the interest nodes and they are added to guarantee the network connectivity. Table 2 lists the paths of four mobile agents.

$$
\begin{aligned}
& I N_{1}=\{6,16,17,27,43,74,78,82,89\} \\
& \ldots \\
& I N_{27}=\{13,19,23,35,39,46,50,52,67,97\} \\
& \ldots \\
& I N_{37}=\{5,37,44,71,74,81,91\} \\
& \ldots \\
& I N_{47}=\{23,42,51,73,75,81,93,97\}
\end{aligned}
$$

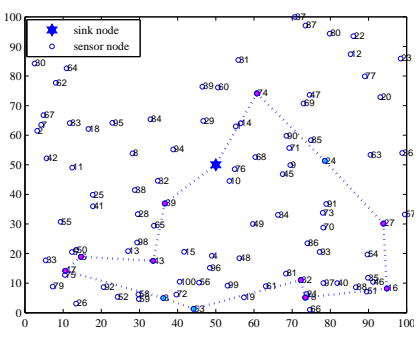

(a)

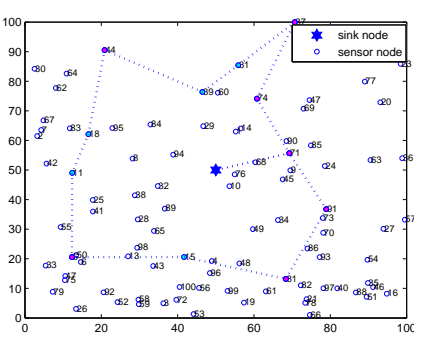

(c)

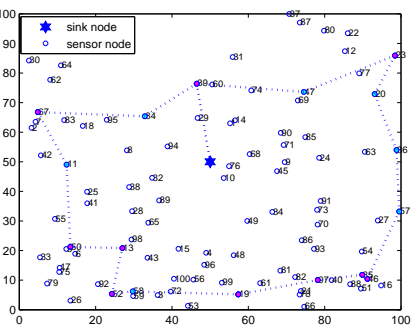

(b)

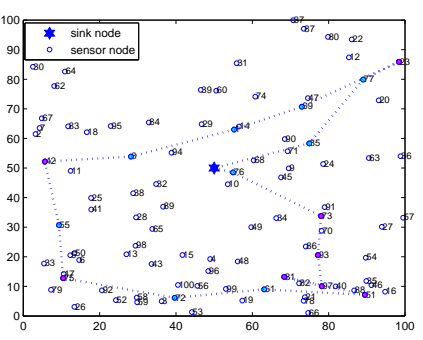

(d)
Fig. 2. The paths designed by MA-Greedy algorithm: (a)The path of mobile agent with ID 1 (b)The path of mobile agent with ID 27 (c)The path of mobile agent with ID 37 (d)The path of mobile agent with ID 47

Table 2: The paths of four mobile agents

\begin{tabular}{cl}
\hline Mobile agent with ID & Paths of mobile agent \\
\hline 1 & $89-43-6-17-3-53-82-78-16-27-24-74$ \\
27 & $39-84-67-11-50-13-52-58-19-97-35-$ \\
37 & $46-57-36-20-23-47-39$ \\
& $71-74-37-31-39-44-18-11-5-15-$ \\
47 & $81-91-71$ \\
& $76-73-93-97-81-51-61-72-75-55-42-$ \\
& $8-1-69-23-77-85$ \\
\hline
\end{tabular}

\section{Experiment results and analysis}

We conduct two experiments to demonstrate the effectiveness of proposed algorithm. In the first experiment, we compare proposed MA-Greedy algorithm with other data gathering algorithms in terms of $C V$. The second experiment shows the results of reconstructing sparse signals by sparse binary matrix. In addition, the performance of signal reconstruction by sparse binary matrix is compared with that by Gaussian random matrix.

\subsection{Experiment Setup}

In the experiment, the threshold $d_{0}$ and communication radius $R$ are set as $50 \mathrm{~m}$ and $30 \mathrm{~m}$, respectively. Because the communication distance between nodes is less than or equal to $R$, free space channel model is used. Experimental parameters are listed in Table 3 .

100 sensor nodes are deployed in the area $100 m \times 100 m$. They are stationary and their locations are known. Fig 3 shows two random uniform layouts of sensor nodes. The location of sink node may have an effect on the balance of energy consumption Safa et al. (2014). Two cases, i.e., sink node is in the center and 
Table 3: Experimental parameters

\begin{tabular}{ll}
\hline parameters & value \\
\hline$R$ & $30 \mathrm{~m}$ \\
$E_{\text {elec }}$ & $1 \mathrm{~J} / \mathrm{bit}$ \\
$E_{\text {amp }}$ & $1 \mathrm{~J} / \mathrm{bit} / \mathrm{m}^{2}$ \\
$n$ & 2 \\
$L$ & $1 \mathrm{bit}$ \\
$d$ & 4 \\
\hline
\end{tabular}

at the corner, are taken into account. In the following experiments, the center and the corner are set at the locations $(50,50)$ and $(0,0)$ of the area in Fig 3 , respectively.

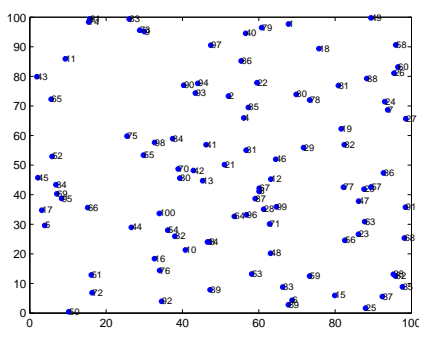

(a)

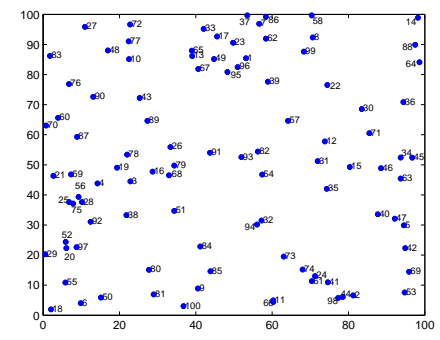

(b)
Fig. 3. Two random uniform layouts of sensor nodes: (a)The first random uniform layout of sensor nodes (b)The second random uniform layout of sensor nodes

According to the two random uniform layouts of sensor nodes and two sink node layouts, we compare $C V$ of the proposed MA-Greedy algorithm with that of the other four data gathering algorithms (i.e. non-CS, plain-CS, Hybrid-CS, and DCSS). In non-CS, the original data(the data without compression) are transmitted through tree-type structure. In plain-CS and Hybrid-CS, the measurement matrix is Gaussian random matrix. However, the measurement matrix in DCSS is sparse binary matrix which is the same as that used in MA-Greedy algorithm.

\subsection{Energy balance performance analysis}

The number $N$ of sensor nodes is set as 100 . If the value of $M$ is smaller, sink node gets fewer measurements and the reconstruction performance will decline. Larger $M$ goes against compressive data gathering. And the value of $M$ affects the energy efficiency of Hybrid-CS. In order to observe the changes of $C V$ in Hybrid-CS and guarantee the sparsity of sparse binary matrix, $M$ is set to a smaller value from the beginning. Furthermore, the measurement matrices in the experiment are Gaussian random matrix and sparse binary matrix. Different measurement matrices can make $C V$ of compressive data gathering algorithms different. So for each value of $M$, we test ten groups of the measurement matrices. The measurement matrices with the minimal $C V$ value are used for compressive data gathering. When sink node is in the center of the area, Fig 4 (a) and Fig 4 (b) depict the changes of $C V$ in the two random uniform layouts, respectively. It can be seen that $C V$ of MA-greedy algorithm is the minimal in the five data gathering algorithms.
The proposed MA-Greedy algorithm uniformly visits each sensor node, which makes total energy consumption of each sensor node is more balanced.

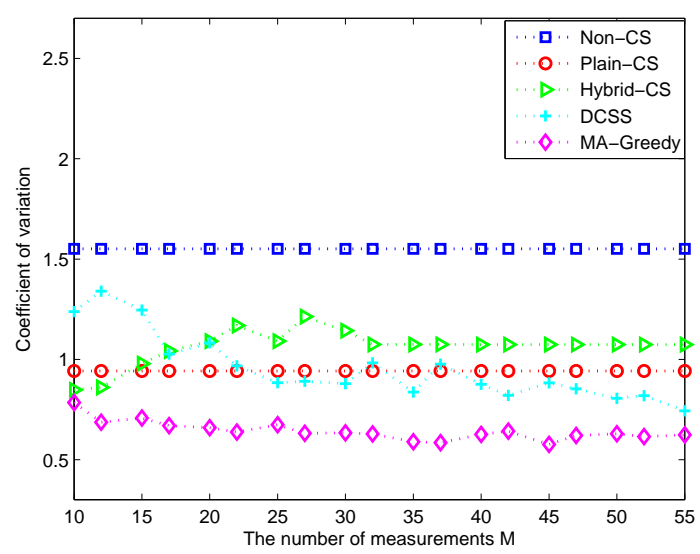

(a)

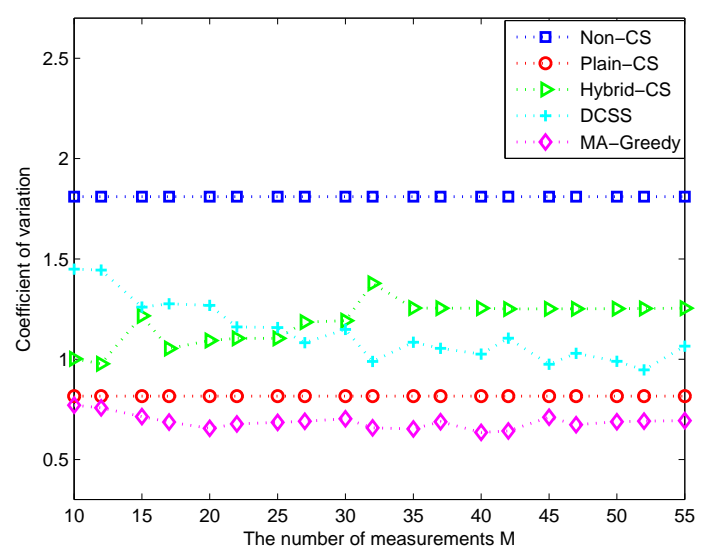

(b)

Fig. 4. The changes of $C V$ with the increase of $M$ for sink node in the center: (a)The first random uniform layout (b)The second random uniform layout

In non-CS, each sensor node transmits the original data to next node along the MST based path. The leaf nodes that don't relay the data from other sensor nodes are far away from sink node. Their data are forwarded to sink node by some intermediate sensor nodes, which makes traffic load of the intermediate sensor nodes heavier, especially sensor nodes around sink node. Total energy consumption between them has a great difference, resulting in larger $C V$. As denoted in two figures, $C V$ of nonCS is larger than that of the other four algorithms, that is, the application of CS to data gathering in WSNs can balance total energy consumption of each sensor node.

In plain-CS, data transmission structure is tree-type. Each sensor node sends or receives $M$ random projections(or weighted sums of random projections). On the condition that tree-type structure is invariable, both total energy consumption $E_{\text {Total }}(i)$ of each sensor node and the average energy consumption $\bar{E}$ in WSNs are proportional to the size of the random projection$\mathrm{s}$ (or weighted sums of random projections). With the increase 
of $M$, the number of random projections(or weighted sums of random projections) increases, which makes $E_{\text {Total }}(i)$ and the standard deviation $\sigma$ increase. However, the increasing ratio of $\bar{E}$ and $E_{\text {Total }}(i)$ is the same. According to Eq.(7), the ratio $\sigma / \bar{E}$ of standard deviation to the average energy consumption can't vary. Seen from Fig 4 (a) and Fig 4(b), $C V$ of plain-CS doesn't change with the value of $M$.

In Hybrid-CS, the sensor nodes that perform CS coding are called aggregators and the others are forwarders. When the value of $M$ is smaller, the number of sensor nodes starting CS coding is more. As shown in Fig 4 when $M$ is less than 15, most sensor nodes are the aggregators. Because the size of random projections(or weighted sums of random projections) transmitted by the aggregators is the same, the difference of total energy consumption among them is less. $C V$ of Hybrid-CS is smaller than that of DCSS. With the increase of $M$, the number of the aggregators reduces, and a majority of forwarders send the original data to corresponding aggregators. Before the original data are transmitted to the aggregators, the data transmission structure is similar to non-CS. For different values of $M$, if the set of aggregators and data transmission structure change less, $C V$ of Hybrid-CS has minor changes. When the value of $M$ changes from 10 to 35 , the set of aggregators between the adjacent $M$ has smaller differences, which causes small fluctuations on the $C V$ curve. When the value of $M$ changes from 35 to 55, the $C V$ curves in two figures are linear. This is because the aggregators and forwarders no longer change when $M$ reaches 35 . With the increase of $M$, the probability that each sensor node is selected for projection nodes becomes higher in DCSS. The $C V$ curves of DCSS roughly degrade in two figures.

The number of measurements $M$ is set as 30 . In accordance with five data gathering algorithms in Fig 4 , Table 4 and Table 5 present the standard deviation $\sigma$, the average energy consumption $\bar{E}$ and $C V$ in two random uniform layouts.

Table 4: $\sigma, \bar{E}$ and $C V$ of five data gathering algorithms in the first random uniform layout

\begin{tabular}{cccccc}
\hline notations & non-CS & plain-CS & Hybrid-CS & DCSS & MA-Greedy \\
\hline$\sigma\left(\times 10^{3} J\right)$ & 0.9127 & 1.6872 & 0.9378 & 6.5424 & 0.8462 \\
$\bar{E}\left(\times 10^{3} J\right)$ & 0.5884 & 1.7909 & 0.8203 & 7.4447 & 1.3355 \\
$C V$ & 1.5512 & 0.9421 & 1.1432 & 0.8788 & 0.6336 \\
\hline
\end{tabular}

Table 5: $\sigma, \bar{E}$ and $C V$ of five data gathering algorithms in the second random uniform layout

\begin{tabular}{cccccc}
\hline notations & non-CS & plain-CS & Hybrid-CS & DCSS & MA-Greedy \\
\hline$\sigma\left(\times 10^{4} J\right)$ & 0.1405 & 0.1537 & 0.1033 & 1.0415 & 0.0924 \\
$\bar{E}\left(\times 10^{4} J\right)$ & 0.0776 & 0.1884 & 0.0867 & 0.9068 & 0.1314 \\
$C V$ & 1.8106 & 0.8158 & 1.1915 & 1.1485 & 0.7032 \\
\hline
\end{tabular}

In order to evaluate the performance of $C V$, Fig[5]a) Fig[5(e) show the total energy consumption layouts of 100 sensor nodes in the first random uniform layout. The comparison result of $\sigma$ between five data gathering algorithms is

$$
\sigma_{d}>\sigma_{b}>\sigma_{c}>\sigma_{a}>\sigma_{e}
$$

where $\sigma_{a}, \sigma_{b}, \sigma_{c}, \sigma_{d}$ and $\sigma_{e}$ are $\sigma$ of five data gathering algorithms in Fig 5 , respectively.

In probability and statistics, $\sigma$ is a measure of the data set dispersion. From Table 4, it can be seen the average energy consumption $\bar{E}$ among different data gathering algorithms is different, so $\sigma$ can't be directly used to evaluate the difference of total energy consumption among sensor nodes. However, $C V$ can eliminate the effect that the difference of $\bar{E}$ has on the difference of the total energy consumption among sensor nodes. The comparison result of $C V$ in Fig 5 is

$$
C V_{a}>C V_{c}>C V_{b}>C V_{d}>C V_{e}
$$

where $C V_{a}, C V_{b}, C V_{c}, C V_{d}$ and $C V_{e}$ are $C V$ of five data gathering algorithms in Fig 5 , respectively.

The total energy consumption of each sensor node is set on an order of magnitude for comparison. In Fig 5 (a) Fig 5 (d), the total energy consumption of a few sensor nodes is significantly larger than that of the others. This is because non-CS, plain-CS and Hybrid-CS use tree-type structure for the data transmission. In the structure, a few sensor nodes send or receive a large number of data, increasing their total energy consumption. In DC$\mathrm{SS}$, there also exist some sensor nodes visited for many times in the shortest path to sink node. In MA-Greedy algorithm, the times each sensor node is visited are almost the same. As shown in Fig 5 (e), the total energy consumption layouts of 100 sensor nodes are more uniform than that in Fig 5 (a) Fig 5 (d).

When sink node is at the corner of the area, the changes of $C V$ corresponding to two random uniform layouts in Fig 3 are depicted in Fig 6(a) and Fig 6 b). When $M$ ranges from 10 to 40 , it can be seen that CS can still make total energy consumption of each sensor node more balanced than non-CS. Because sink node is at the corner, a mass of data flock to the sensor nodes around sink node, which increases their total energy consumption. Compared with sink node layout in Fig 5, sink node layout in Fig 6 is more affected by the boundary effect. In compressive data gathering, the random projections(or the weighted sums of the random projections) must get through sensor nodes around sink node. Meanwhile, the number of these sensor nodes is less than that without the boundary effect, and their total energy consumption increases.

In Hybrid-CS, sensor nodes closer to sink node must be the aggregators. They forward the data from the forwarders and other aggregators. With the increase of $M$, their total energy consumption is more relative to the forwarders far away from sink node. Total energy consumption among sensor nodes is more imbalanced and the value of $C V$ gets larger. Overall, $C V$ curves in two figures demonstrates upgrade tendency with the increase of $M$. When the value of $M$ ranges from 10 to 30, larger fluctuations appear on the $C V$ curves of Hybrid-CS. To be specific, only sensor nodes which make the current energy consumption in WSNs less than the foregoing energy consumption become the aggregators. For different values of $M$, the aggregators and forwarders change. When the set of aggregators between the adjacent $M$ and data transmission structure have large differences, this will cause larger fluctuations on the $C V$ curves. 


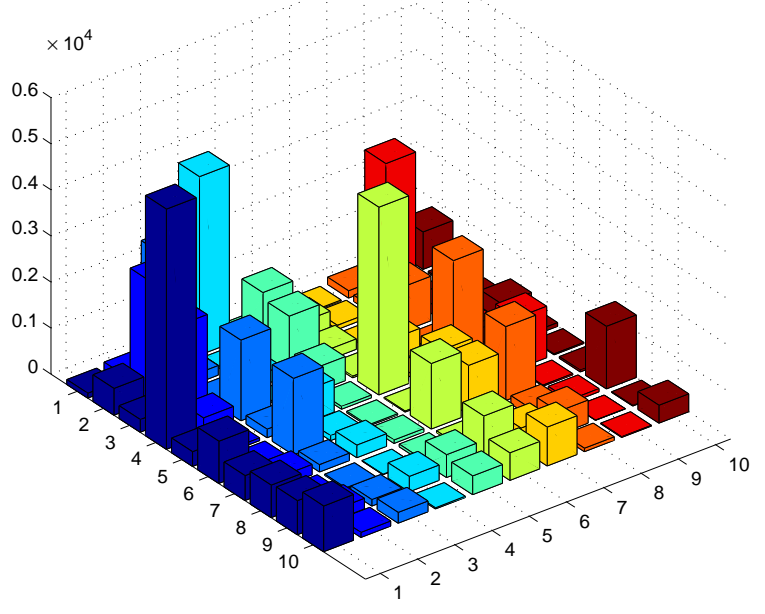

(a)

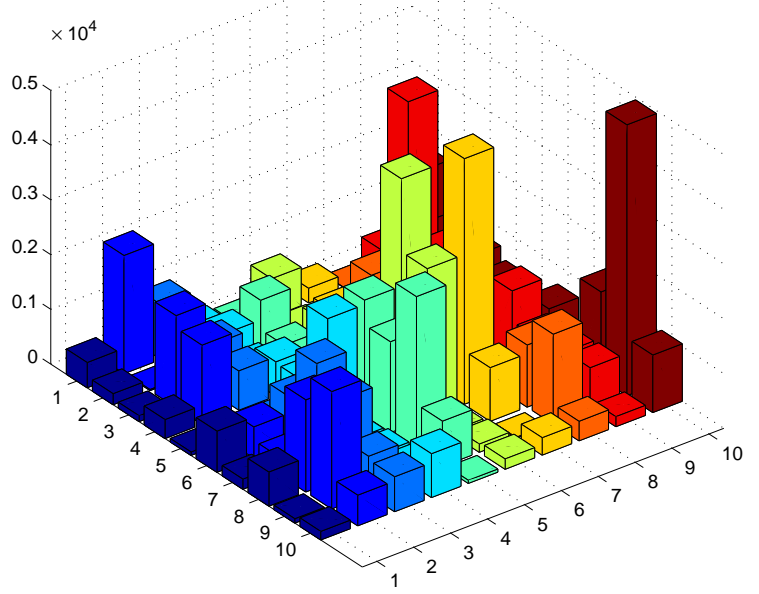

(c)

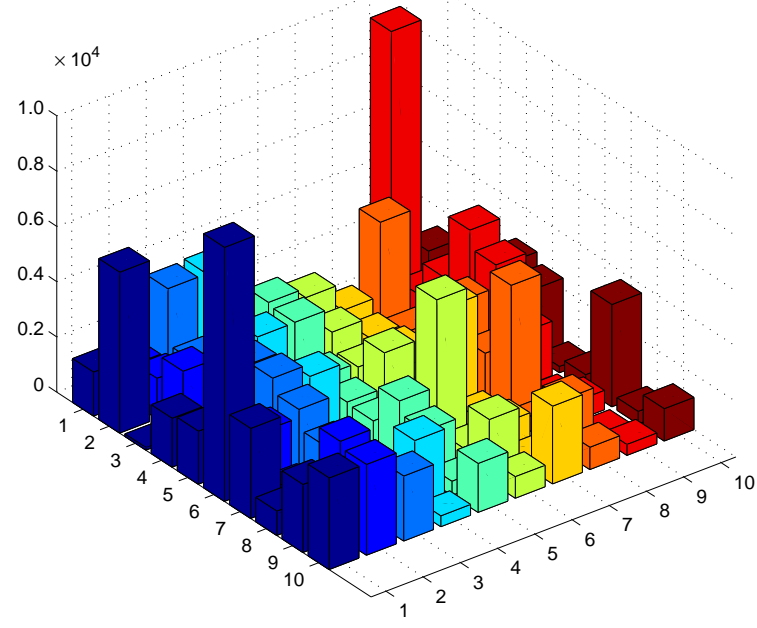

(b)

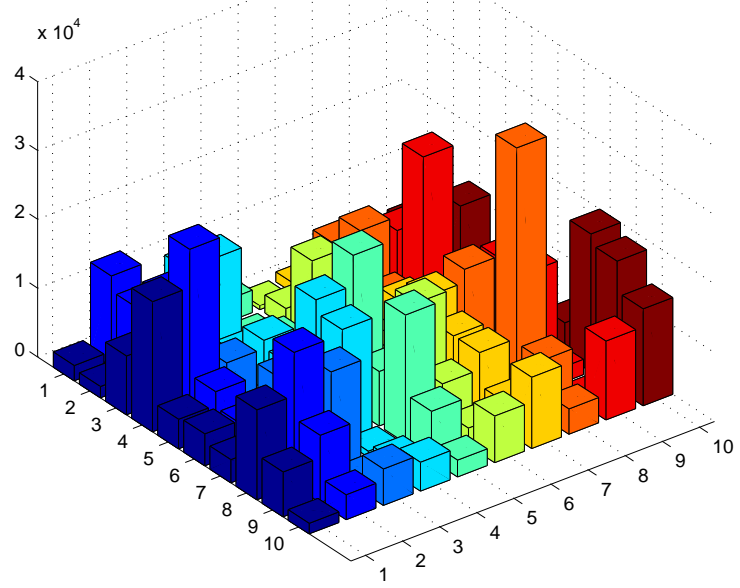

(d)

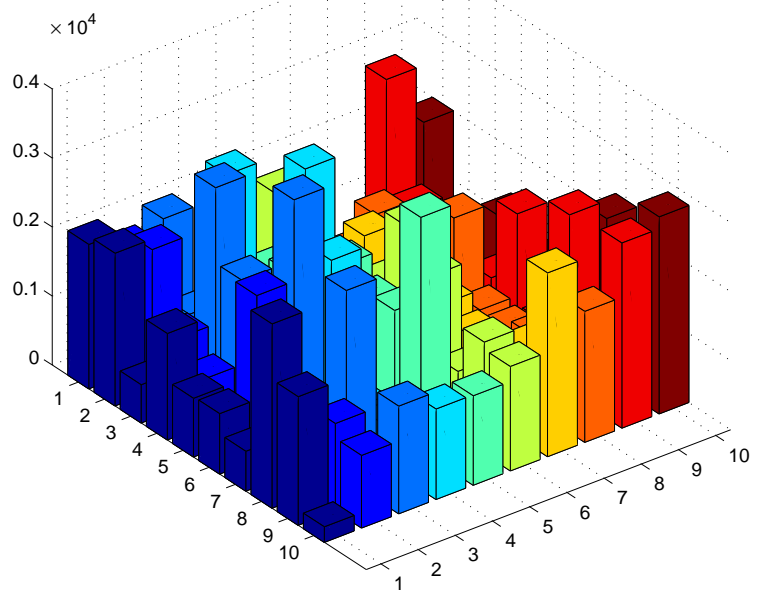




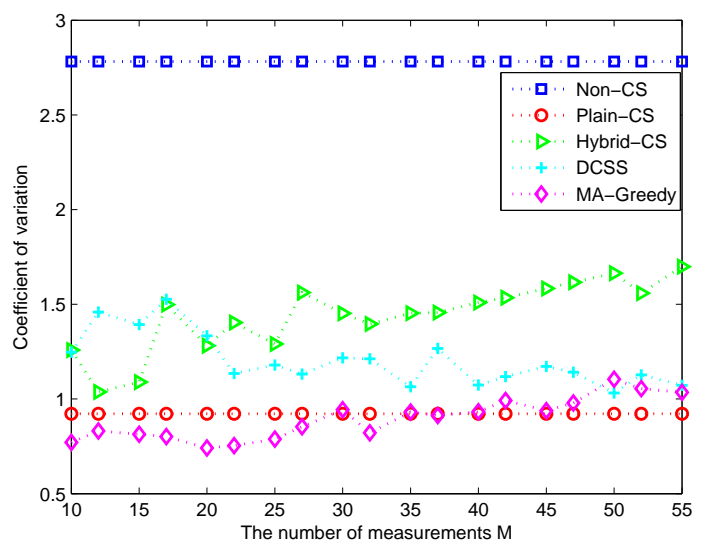

(a)

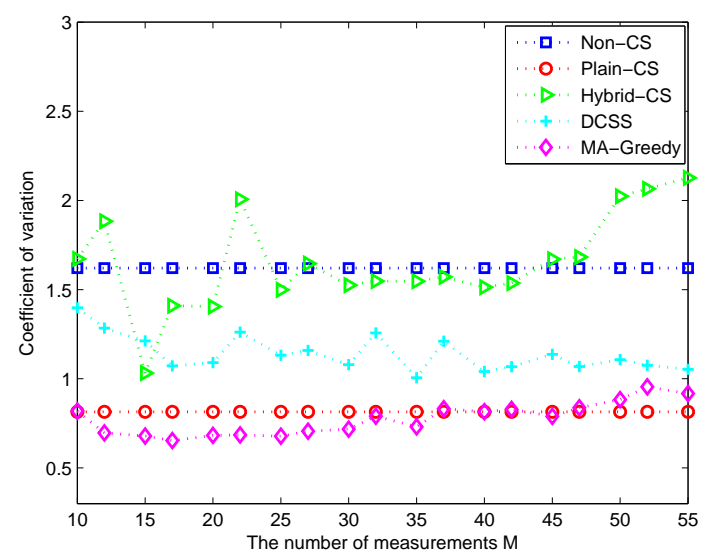

(b)

Fig. 6. The changes of $C V$ with the increase of $M$ for sink node at the corner: (a)The random uniform layout (b)The second random uniform layout

In DCSS, the interest nodes transmit the random projection$\mathrm{s}$ to the appointed projection nodes through the shortest path. After receiving all the random projections, the projection nodes send them to sink node through the shortest path. The aggregation isn't considered in the shortest path. Some sensor nodes can be used repeatedly for many times. The phenomenon that appears in Hybrid-CS also exists in DCSS. However, each sensor node transmits the same number of random projection$\mathrm{s}$ (or the weighed sums of random projections) in plain-CS. The $C V$ of plain-CS is smaller than that of the two CDG algorithms(DCSS and Hybrid-CS), as depicted in Fig 6 a) and Fig 6b). Besides that, Hybrid-CS requires to determine the aggregators and forwarders. Its $C V$ is easily affected by the locations of sink node and the value of $M$.

The proposed MA-Greedy algorithm needn't choose the projection nodes. When $M$ is less than $40, C V$ of MA-Greedy algorithm is smaller than that of the other four algorithms. That is, total energy consumption of each sensor node is more balanced. But sink node is at the corner, the interest nodes are far away from it. With the increase of $M$, mobile agent returns to sink none by the more sensor nodes which aren't the interest nodes. Sensor node close to sink node can be repeatedly visited for many times. When $M$ increases, $C V$ of MA-Greedy algorithm is larger than that of plain-CS. Total energy consumption of each sensor node appears imbalanced. $M$ is set as 25 , Table 6 and Table 7 present the standard deviation $\sigma$, the average energy consumption $\bar{E}$ and $C V$ in two random uniform layouts.

Table 6: $\sigma, \bar{E}$ and $C V$ of five data gathering algorithms in the first random uniform layout

\begin{tabular}{cccccc}
\hline notations & non-CS & plain-CS & Hybrid-CS & DCSS & MA-Greedy \\
\hline$\sigma\left(\times 10^{4} J\right)$ & 0.3400 & 0.1405 & 0.1152 & 1.2990 & 0.1116 \\
$\bar{E}\left(\times 10^{4} J\right)$ & 0.1222 & 0.1524 & 0.0892 & 1.1014 & 0.1416 \\
$C V$ & 2.7823 & 0.9219 & 1.2915 & 1.1794 & 0.7881 \\
\hline
\end{tabular}

Table 7: $\sigma, \bar{E}$ and $C V$ of five data gathering algorithms in the second random uniform layout

\begin{tabular}{cccccc}
\hline notations & non-CS & plain-CS & Hybrid-CS & DCSS & MA-Greedy \\
\hline$\sigma\left(\times 10^{4} J\right)$ & 0.2169 & 0.1282 & 0.1225 & 1.1912 & 0.0831 \\
$\bar{E}\left(\times 10^{4} J\right)$ & 1.1338 & 0.1573 & 0.1000 & 1.0528 & 0.1499 \\
$C V$ & 1.6211 & 0.8150 & 1.4990 & 1.1315 & 0.6784 \\
\hline
\end{tabular}

Take the first random uniform layout as an example. The total energy consumption layouts of 100 sensor nodes are shown in Fig7(a) Fig 7(e). The comparison result of $C V$ is

$$
C V_{a}>C V_{c}>C V_{d}>C V_{b}>C V_{e}
$$

where $C V_{a}, C V_{b}, C V_{c}, C V_{d}$ and $C V_{e}$ are $C V$ of five data gathering algorithms in Fig 7 respectively. $C V$ of MA-Greedy algorithm is the minimum in five data gathering algorithms.

In Fig 7 (a) and Fig 7 (c), there exists sensor node with ID 16 in the position $(2,6,0)$. Seen from Fig $3(a)$, the sensor node is close to sink node at the corner. Due to tree-type structure, it needs to forward the data from other sensor nodes. Its total energy consumption is larger than others. Plain-CS also uses the similar tree-type structure, so the total energy consumption of the sensor node with ID 16 is the second largest in Fig 7 b). The distance between sensor node with ID 5 and sink node is $29.8703 m$, which is close to $R$. Its total energy consumption is the largest in Fig 7(e). For DCSS in Fig 7(d), each projection nodes transmit random projection from interest nodes through the shortest path. Sensor node with ID 5 is in the shortest path to sink node, and its total energy consumption is larger than the great majority of sensor nodes.

From the above, when sink node is in the center of the area, the proposed MA-Greedy algorithm precedes the other four data gathering algorithms in terms of energy balance. If sink node is at the corner of the area, MA-Greedy algorithm has an advantage for smaller $M$.

\subsection{Reconstruction performance of sparse signals}

Suppose $k$ burst events happen, they are detected by $k$ sensor nodes. The data readings of the $k$ sensor nodes are one and the data readings of other sensor nodes are zero. The data readings 


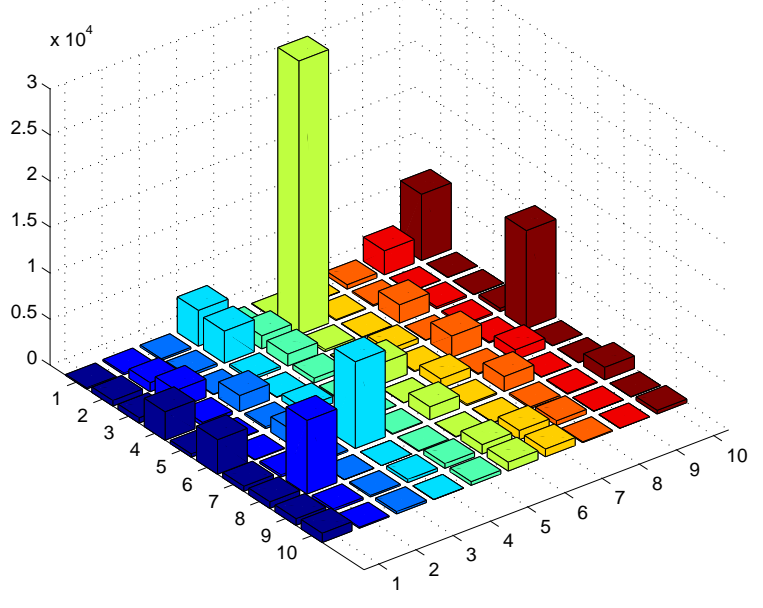

(a)

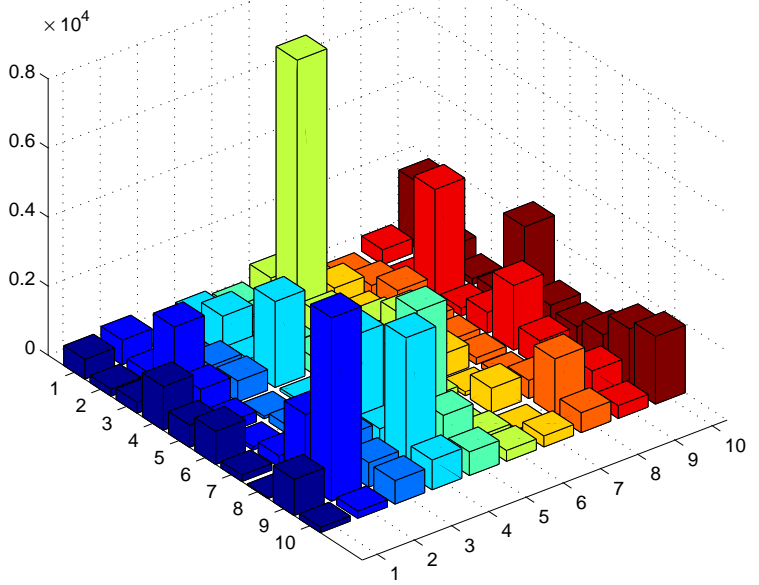

(c)

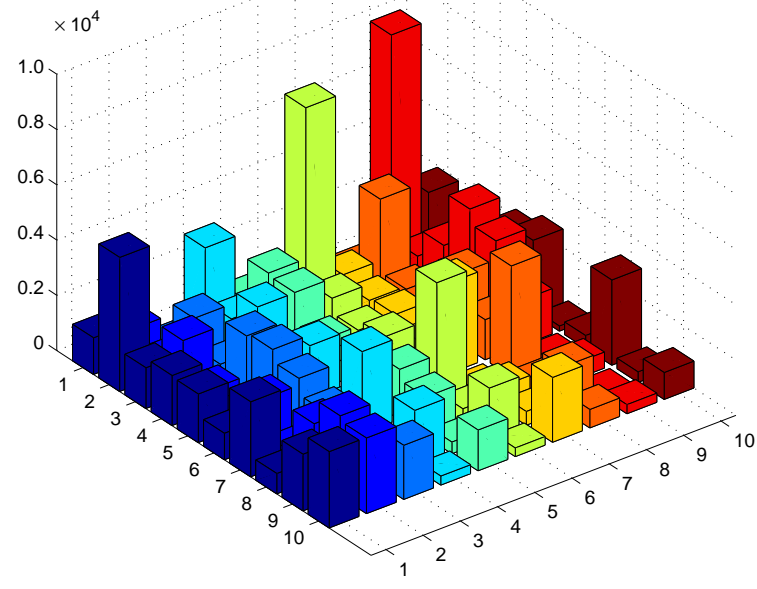

(b)

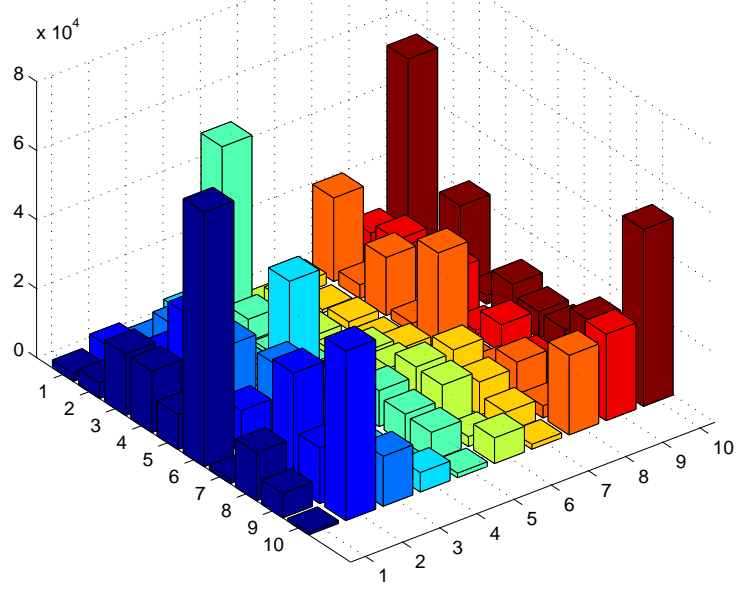

(d)

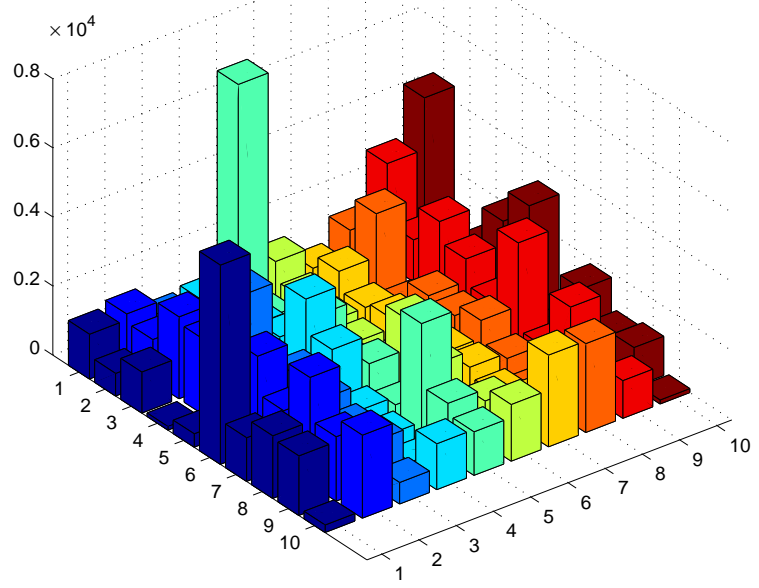

11

(e)

Fig. 7. Energy consumption of each sensor node for sink node at the corner: (a)non-CS (b)plain-CS (c)Hybrid-CS (d)DCSS (e)MA-Greedy 
$x$ of all the sensor nodes are $k$ sparse zero-one signals. The signals can be sampled through the measurement matrix in CS. In the experiment, the measurement matrices are Gaussian random matrix and sparse binary matrix, respectively. $\Psi$ is identity matrix. BP is used to reconstruct the signals Berinde et al. (2008); Indyk (2010).

Relative error $\varepsilon$ of reconstructing the signals is defined in Eq.(13).

$$
\varepsilon=\frac{\|x-\hat{x}\|_{2}}{\|x\|_{2}}
$$

where $x$ are $k$ sparse signals and $\hat{x}$ are the reconstructed signals. If $\varepsilon \leqslant 10^{-4}$, we think the signals can be accurately reconstructed. In the following figures, we also draw the percentage of accurately reconstructing $x$.
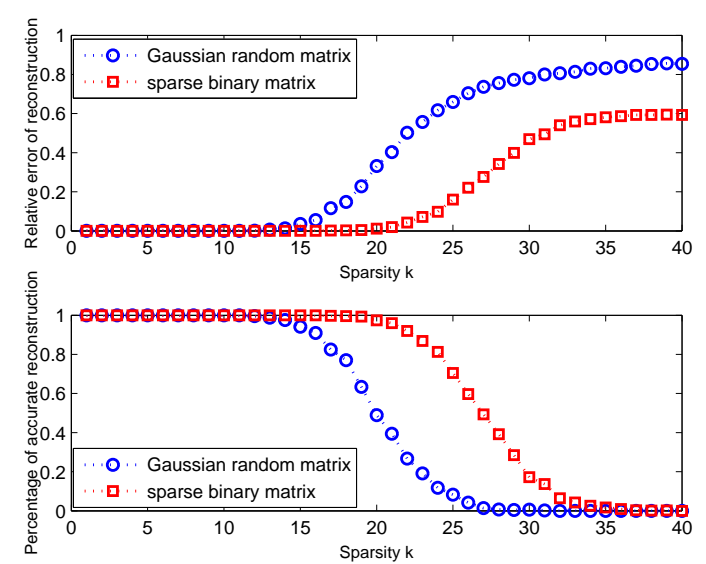

(a)

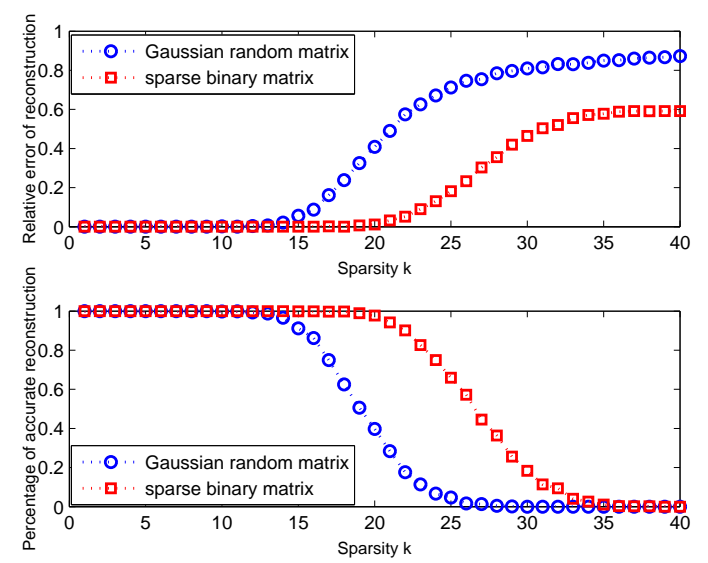

(b)

Fig. 8. Relative error and percentage of accurate reconstruction versus sparsity $k$ : (a)sink node in the center (b) sink node at the corner

When the number $N$ of sensor nodes is 100 and the value of $M$ is 50, 1000 zero-one signals $x$ which are $k$ sparse are tested. When sink node is in the center and at the corner, Fig 8 a) and Fig $[$ b) denote the results of reconstructing $x$ by Gaussian random matrix and sparse binary matrix used in the first random uniform layout, respectively. When $k$ ranges from 1 to $15, k$ sparse zero-one signals $x$ can be accurately reconstructed by the

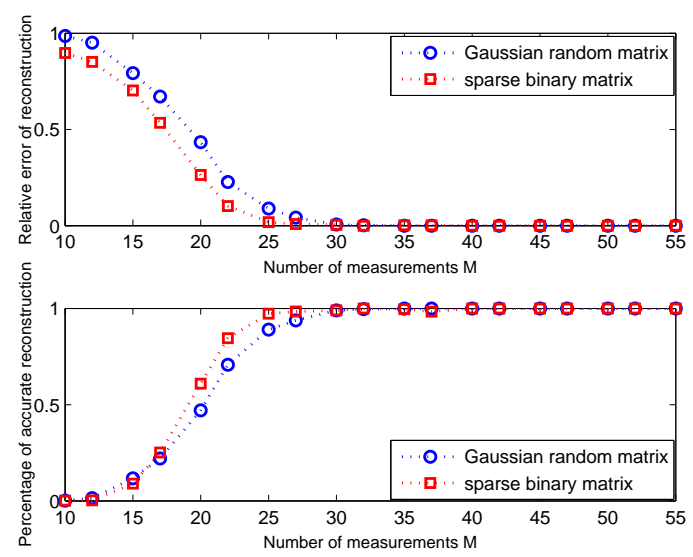

(a)

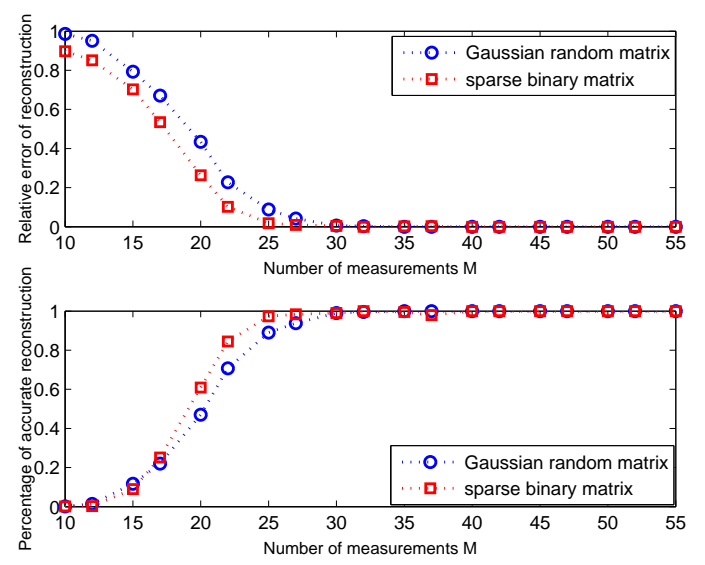

(b)

Fig. 9. Relative error and percentage of accurate reconstruction versus the number $M$ of measurements: (a) sink node in the center (b) sink node at the corner

two classes of measurement matrices. Seen from Fig 8 , with the increase of $k$, the relative error $\varepsilon$ of reconstructing $x$ by sparse binary matrix is smaller than that by Gaussian random matrix. The smaller $\varepsilon$ is, the larger the percentage of accurately reconstructing $x$ is. When $k$ ranges from 15 to 40 , the percentage of accurately reconstructing $x$ becomes low gradually. Because the value of $M$ is fixed, the smaller $k$ is enough for accurately reconstructing $x$. But $k$ sparse zero-one signals $x$ with the larger $k$ require more measurements for accurate reconstruction. With the increase of $k, \varepsilon$ increases and the percentage of accurate reconstruction descends.

When $k$ is 5 and the number $M$ of measurements changes from 10 to 55, the results of reconstructing sparse zero-one signals $x$ are shown in Fig 9 , where the measurement matrices are corresponding to that in Fig 8 . When $M$ ranges from 10 to 30, it can be seen that relative error $\varepsilon$ of reconstructing $x$ gradually becomes smaller. In Fig 9 (a), relative error $\varepsilon$ of reconstructing $x$ by Gaussian random matrix increases by $0.0024 \sim 0.1707$ than that by sparse binary matrix, and the percentage of accurate reconstruction descends by $0.027 \sim 0.1390$. When $M$ is within the range of $30 \sim 55, x$ can be accurately reconstructed by two classes of measurement matrices. Similar to Fig 9 (a), Fig 9 (b) 
shows the relative error $\varepsilon$ of reconstructing $x$ by Gaussian random matrix increases by $0.003 \sim 0.1707$ than that by sparse binary matrix, and the percentage of accurate reconstruction decreases by $0.027 \sim 0.1390$. In other words, the curves in Fig 9 a) and Fig 9 (b) are almost the same.

As can be seen from Fig 8 and Fig 9 the reconstruction quality by sparse binary matrix is comparable to that by Gaussian random matrix. Under the same conditions, the percentage of accurately reconstructing sparse zero-one signals $x$ by sparse binary matrix is larger than that by Gaussian random matrix. For energy-efficient WSNs, sparse binary matrix is an alternative to Gaussian random matrix for data gathering.

\section{Conclusions}

The paper studies compressive data gathering in WSNs. Taking into account dynamic change of practical network topology and the feature of mobile agents, we propose a mobile agent based compressive data gathering algorithm(MA-Greedy). The algorithm considers the relation between sparse binary matrix and data gathering. In the best-case scenario, the average time complexity of MA-Greedy algorithm is $O\left(M \tau_{\text {max }} \log \tau_{\text {max }}\right)$, where $\tau_{\text {max }}\left(\tau_{\text {max }} \leq N\right)$ is the maximal number of the interest nodes in each row of sparse binary matrix. In the worst-case scenario, the time complexity is $O\left(M N^{3}\right)$. We conduct the experiments in random uniform layouts of sensor nodes to verify the effectiveness of MA-Greedy algorithm. The experiment results show MA-Greedy algorithm can improve the balance of energy consumption. What's more, the results of reconstructing sparse zero-one signals by sparse binary matrix are better than that by Gaussian random matrix.

\section{Acknowledgment}

This work of the paper is supported by National Science Foundations of China(No.61174016) and the Natural Science Foundation of Hebei Province of China(No.F2015202214).

\section{References}

Abdulla, A. E., Nishiyama, H., Kato, N., 2012. Extending the lifetime of wireless sensor networks: A hybrid routing algorithm. Computer Communications 35 (9), 1056-1063.

Anastasi, G., Conti, M., Di Francesco, M., Passarella, A., 2009. Energy conservation in wireless sensor networks: A survey. Ad hoc networks 7 (3), 537-568.

Bajwa, W. U., Haupt, J. D., Sayeed, A. M., Nowak, R. D., 2007. Joint sourcechannel communication for distributed estimation in sensor networks. IEEE Transactions on Information Theory 53 (10), 3629-3653.

Baraniuk, R. G., 2007. Compressive sensing. IEEE signal processing magazine 24 (4).

Berinde, R., Gilbert, A. C., Indyk, P., Karloff, H., Strauss, M. J., 2008. Combining geometry and combinatorics: A unified approach to sparse signal recovery. In: 2008 46th Annual Allerton Conference on Communication, Control, and Computing. IEEE, pp. 798-805.

Candès, E. J., 2006. Compressive sampling. In: Proceedings of the international congress of mathematicians. Vol. 3. Madrid, Spain, pp. 1433-1452.

Chen, M., Gonzalez, S., Leung, V., 2007. Applications and design issues for mobile agents in wireless sensor networks. IEEE Wireless Communications 14 (6), 20-26.
Chen, W., Wassell, I. J., 2011. Energy efficient signal acquisition via compressive sensing in wireless sensor networks. In: 2011 6th International Symposium on Wireless and Pervasive Computing (ISWPC). IEEE, pp. 1-6.

Donoho, D. L., 2006. Compressed sensing. IEEE Transactions on Information Theory 52 (4), 1289-1306.

Fazel, F., Fazel, M., Stojanovic, M., 2011. Random access compressed sensing for energy-efficient underwater sensor networks. IEEE Journal on Selected Areas in Communications 29 (8), 1660-1670.

Heinzelman, W. B., Chandrakasan, A. P., Balakrishnan, H., 2002. An application-specific protocol architecture for wireless microsensor networks. IEEE Transactions on Wireless Communications 1 (4), 660-670.

Indyk, P., 2010. Sparse Recovery Using Sparse Random Matrices. Springer Berlin Heidelberg.

Jafarpour, S., Xu, W., Hassibi, B., Calderbank, R., 2009. Efficient and robust compressed sensing using optimized expander graphs. IEEE Transactions on Information Theory 55 (9), 4299-4308.

Lee, S., Pattem, S., Sathiamoorthy, M., Krishnamachari, B., Ortega, A., 2009. Compressed sensing and routing in multi-hop networks. University of Southern California CENG Technical Report.

Li, S., Qi, H., 2013. Distributed data aggregation for sparse recovery in wireless sensor networks. In: 2013 IEEE International Conference on Distributed Computing in Sensor Systems (DCOSS). IEEE, pp. 62-69.

Luo, C., Wu, F., Sun, J., Chen, C. W., 2009. Compressive data gathering for large-scale wireless sensor networks. In: Proceedings of the 15th annual international conference on Mobile computing and networking. ACM, pp. $145-156$.

Mamaghanian, H., Khaled, N., Atienza, D., Vandergheynst, P., 2011. Compressed sensing for real-time energy-efficient ecg compression on wireless body sensor nodes. IEEE Transactions on Biomedical Engineering 58 (9), 2456-2466.

Qi, H., Wang, F., 2001. Optimal itinerary analysis for mobile agents in ad hoc wireless sensor networks. Proceedings of the IEEE, 147-153.

Quer, G., Masiero, R., Munaretto, D., Rossi, M., Widmer, J., Zorzi, M., 2009. On the interplay between routing and signal representation for compressive sensing in wireless sensor networks. In: Information Theory and Applications Workshop, 2009. IEEE, pp. 206-215.

Safa, H., El-Hajj, W., Zoubian, H., 2014. A robust topology control solution for the sink placement problem in wsns. Journal of Network and Computer Applications 39, 70-82.

Wang, W., Garofalakis, M., Ramchandran, K., 2007. Distributed sparse random projections for refinable approximation. In: Proceedings of the 6th international conference on Information processing in sensor networks. ACM, pp. 331-339.

West, D. B., West, D. B., 2001. Introduction to graph theory - second edition. P-decomposable, 57 factorisable, 4 over P, 43 (4), 263-277.

Xiang, L., Luo, J., Rosenberg, C., 2013. Compressed data aggregation: Energyefficient and high-fidelity data collection. IEEE/ACM Transactions on Networking 21 (6), 1722-1735.

Xie, M., Han, S., Tian, B., Parvin, S., 2011. Anomaly detection in wireless sensor networks: A survey. Journal of Network and Computer Applications 34 (4), 1302-1325.

Xu, W., Hassibi, B., 2007. Further results on performance analysis for compressive sensing using expander graphs. In: Conference Record of the FortyFirst Asilomar Conference on Signals, Systems and Computers, 2007. ACSSC 2007. IEEE, pp. 621-625.

Yick, J., Mukherjee, B., Ghosal, D., 2008. Wireless sensor network survey. Computer networks 52 (12), 2292-2330.

Zhao, S. f., Huang, L. s., 2012. One compressive sensing algorithm based on local data in wsn. Journal of Fudan University (Natural Science) 6, 014. 